\title{
Novas ocorrências de liquens corticícolas crostosos para a região sul do Brasil
}

\author{
Márcia Isabel Käffer ${ }^{1,2,5}$, Marcela Eugenia da Silva Cárceres ${ }^{3}$, Vera Maria Ferrão Vargas ${ }^{1,4}$ e Suzana Maria de Azevedo Martins ${ }^{2}$ \\ Recebido em 23/12/2009. Aceito em 19/08/2010 \\ RESUMO - (Novas ocorrências de liquens corticícolas crostosos para a região sul do Brasil). São apresentadas 26 novas espécies de liquens crostosos para \\ o Brasil e Estado do Rio Grande do Sul, sendo três destas novas ocorrências também para o Brasil e 23 novos registros para o Estado. Os liquens foram \\ analisados em 300 forófitos distribuídos em 32 bairros da cidade de Porto Alegre e na área do Parque Estadual de Itapuã, Viamão, RS. \\ Palavras-chave: distribuição, forófitas, fungos liquenizados, Porto Alegre, RS
}

ABSTRACT - (New records of corticolous crustose lichens from southern Brazil). Twenty six new records of corticolous crustose lichen species for Brazil and Rio Grande do Sul state are presented; three are new records for Brazil and 23 new records for the state. Lichens were analyzed on 300 host trees distributed in 32 districts of the city of Porto Alegre and Itapuã State Park, Viamão, RS.

Key words: distribution, host trees, lichenized fungi, Porto Alegre, RS

\section{Introdução}

Os liquens fazem parte de um grupo extremamente diverso, variando em sua complexidade, desde formas muito simples até estruturas morfológicas e anatômicas muito complexas. São separados em formas ou tipos, entre os principais estão os crostosos, esquamulosos, foliosos, filamentosos e fruticosos. Os fungos liquenizados crostosos, também denominados microliquens não possuem córtex inferior e aderem-se ao substrato por toda sua superfície inferior através das hifas da medula (Nash 1996; Marcelli 2006). Aproximadamente $75 \%$ dos liquens formam talos crostosos (Ahmadjian 1993). Entretanto, ainda são poucos os estudos que contemplem os liquens crostosos no Brasil.

Marcelli (2008) apresenta 2874 espécies liquênicas, sendo que $49,4 \%$ pertencem ao grupo dos fungos liquenizados crostosos. Cáceres (2007) em levantamento realizado em vários tipos de ecossistemas na região nordeste do Brasil registrou 437 táxons crostosos, dos quais 18 foram mencionados como novos para a ciência, e 14 novas combinações. Dal Forno (2009) em trabalho realizado com a família Graphidaceae em vegetação de restinga, na região sul do Paraná, relacionou 57 espécies, sendo oito novas para a ciência e 44 novas ocorrências para o estado do Paraná. Para o Rio Grande do Sul já foram registrados 412 táxons crostosos (Spielmann 2006). Ainda há contribuições de Martins (2006) com citação de 43 táxons crostosos, dos quais três constituíam-se na época, em novos gêneros, 20 espécies como novos registros para o Estado e sete novas espécies para o Brasil.

O presente trabalho tem por objetivo apresentar as novas ocorrências de liquens corticícolas crostosos para o Brasil e estado do Rio Grande do Sul. As espécies liquênicas aqui apresentadas foram registradas no âmbito de um trabalho mais abrangente, de estudo da micota liquenizada como bioindicadores da qualidade do ar, em área urbana da cidade de Porto Alegre, RS, Brasil.

\section{Material e métodos}

Área de estudo - O trabalho foi realizado na cidade de Porto Alegre, capital do estado do Rio Grande do Sul, Brasil, que abrange uma área de 502,5 $\mathrm{km}^{2}$, está localizada na região da Depressão Central, entre as coordenadas $30^{\circ} 01 ' 53^{\prime \prime}$ de latitude sul e $51^{\circ} 13^{\prime} 19^{\prime \prime}$ de longitude oeste, às margens do lago Guaíba. O município está inserido nas unidades geomorfológicas do Escudo-riograndense e da Depressão Central, além de sofrer influência da Planície Costeira (Vieira, 1984). O clima é subtropical úmido apresentando temperatura média anual de $19,4^{\circ} \mathrm{C}$, umidade relativa média do ar de $76 \%$ e índice pluviométrico de $1.324 \mathrm{~mm}$ anuais (Livi, 1998).

Amostragem e identificação - Os liquens foram coletados em 300 forófitas distribuídos em praças, parques e/ou ruas, em 32 bairros da cidade e no Parque Estadual de Itapuã, localizado no distrito de Viamão (50 $50^{\prime}$ e $51^{\circ}$ $05^{\prime} \mathrm{W}$ e $30^{\circ} 20^{\prime}$ e $30^{\circ} 27^{\prime} \mathrm{S}$ ) distante $57 \mathrm{~km}$ de Porto Alegre, sendo este denominado estação controle. As atividades de campo ocorreram entre o período de julho de 2007 a junho de 2008.

As identificações foram realizadas com auxílio de microscópio estereoscópico e óptico, através de secções anatômicas dos talos e frutificações. Testes de coloração através de hidróxido de potássio $20 \%$ (KOH) e lugol (reação I) para determinar presença de substâncias e/ou reações no córtex, himênio, asco e ascósporos foram realizados quando necessário. A identificação foi baseada nas seguintes bibliografias: Aptroot (2008), Cáceres (2007), Dal Forno (2009), Lücking \& Rivas-Plata (2008), Lücking et al. (2008, 2009), Rivas Plata et al. (2009) e Sipman (2006). O material coletado se encontra incorporado ao Herbário Prof. Dr. R.H. Alarich Schultz (HAS) do Museu de Ciências Naturais da Fundação Zoobotânica do Rio Grande do Sul.

\section{Resultados e discussão}

Foram registradas 26 espécies de liquens crostosos, distribuídos em 10 famílias com 15 gêneros, sendo três novas ocorrências para o Brasil: Graphis dolichographa Nyl., Phaeographis intricans (Nyl.) Staiger e Pyrenula

\footnotetext{
1 Universidade Federal do Rio Grande do Sul, Curso de Pós-Graduação em Ecologia, RS, Brasil

2 Fundação Zoobotânica do Rio Grande do Sul, Museu de Ciências Naturais, Porto Alegre, RS, Brasil

Universidade Federal de Sergipe, Departamento de Biociências, Itabaiana, SE, Brasil

Fundação Estadual de Proteção Ambiental, Porto Alegre, RS, Brasil

Autor de correspondência: m.kaffer@terra.com.br
} 
mucosa (Vain.) R. C. Harris, e 23 novos registros para o Rio Grande do Sul: Anisomeridium tamarindii (Fée) R. C. Harris, Bacidia russeola (Kremp.) Zahlbr., Bactrospora myriadea (Fée) Egea \& Torrente, Chapsa cinchonarum (Fée) A. Frisch, Coenogonium subdilutum (Malme) Lücking, Aptroot \& Sipman, Cratiria lauricassiae (Fée) Marbach, Enterographa compunctula (Nyl.) Redinger, Fissurina instabilis (Nyl.) Nyl., Graphis archerii Dal-Forno \& Eliasaro, Graphis geraensis Redinger, Graphis kakaduensis A. W. Archer, Graphis parallela (Müll. Arg.) Cáceres \& Lücking, Graphis paraserpens Lizano \& Lücking, Graphis rigidula Müll. Arg., Graphis schiffneri Zahlbr, Graphis submarginata Lücking, Haematomma personii (Fée) A. Massal., Lecanora caesiorubella Ach., Malcolmiella vinosa (Eschw.) Kalb \& Lücking, Pertusaria carneola (Eschw.) Müll. Arg., Pertu- saria wulfenioides De Lesd., Phaeographis punctiformis (Eschw.) Müll. Arg. e Pyrenula pyrenuloides (Mont.) R. C. Harris. Na tabela 1 se encontra a relação das espécies, as novas ocorrência para o país e/ou Estado, a distribuição geográfica, assim como o local onde o espécime foi registrado.

O maior número de espécies (13) pertence à família Graphidaceae, seguida dos exemplares de Lecanoraceae, Pertusariaceae e Pyrenulaceae com dois representantes cada. Os forófitos onde os liquens foram coletados pertencem às espécies: Brachychyton populneum (Schott \& Endl.) R. Br., Enterolobium contortiisiliquum (Vell.) Morong, Hovenia dulcis Thunb., Ligustrum japonicum Thunb., Melia azedarach L., Myrsine umbellata Mart., Peltotphorum dubium (Spreng.) Taub. e Tabebuia heptaphylla (Vell.) Toledo.

Tabela 1. Relação das novas ocorrências das espécies liquênicas registradas nas áreas de estudo. Legenda: RS = Rio Grande do Sul

\begin{tabular}{|c|c|c|c|c|}
\hline \multirow{2}{*}{ Família/Espécies } & \multirow{2}{*}{$\begin{array}{c}\text { Nova } \\
\text { ocorrência }\end{array}$} & \multicolumn{2}{|c|}{ Localização } & \multirow{2}{*}{ Distribuição geográfica } \\
\hline & & Porto Alegre & Viamão & \\
\hline \multicolumn{5}{|l|}{ Coenogoniaceae } \\
\hline Coenogonium subdilutum (Malme) Lücking, Aptroot \& Sipman & RS & $\mathrm{x}$ & $\mathrm{x}$ & Neotropical \\
\hline \multicolumn{5}{|l|}{ Graphidaceae } \\
\hline Chapsa cinchonarum (Fée) A. Frisch & RS & $\mathrm{x}$ & $\mathrm{x}$ & Pantropical \\
\hline Graphis archerii Dal-Forno \& Eliasaro & RS & $\mathrm{x}$ & $\mathrm{x}$ & \\
\hline Graphis dolichographa Nyl. & Brasil & $\mathrm{x}$ & & $\begin{array}{c}\text { Neotropical e } \\
\text { Paleotropical Oriental }\end{array}$ \\
\hline Graphis geraensis Redinger & RS & $\mathrm{x}$ & $\mathrm{x}$ & Pantropical \\
\hline Graphis kakaduensis A. W. Archer & $\mathrm{RS}$ & $\mathrm{x}$ & $\mathrm{x}$ & Pantropical \\
\hline Graphis parallela (Müll. Arg.) Cáceres \& Lücking & $\mathrm{RS}$ & $\mathrm{x}$ & & Pantropical \\
\hline Graphis paraserpens Lizano \& Lücking & $\mathrm{RS}$ & $\mathrm{x}$ & & Pantropical \\
\hline Graphis rigidula Müll. Arg. & RS & $\mathrm{x}$ & $\mathrm{x}$ & Neotropical \\
\hline Graphis schiffneri Zahlbr & RS & $\mathrm{x}$ & $\mathrm{x}$ & Pantropical \\
\hline Graphis submarginata Lücking & $\mathrm{RS}$ & $\mathrm{x}$ & & Pantropical \\
\hline Fissurina instabilis (Nyl.) Nyl. & $\mathrm{RS}$ & $\mathrm{x}$ & & Pantropical \\
\hline Phaeographis intricans (Nyl.) Staiger & Brasil & $\mathrm{x}$ & & Pantropical e Temperada \\
\hline Phaeographis punctiformis (Eschw.) Müll. Arg. & RS & & $\mathrm{x}$ & Pantropical \\
\hline \multicolumn{5}{|l|}{ Lecanoraceae } \\
\hline Haematomma personii (Fée) A. Massal. & RS & $\mathrm{x}$ & $\mathrm{x}$ & Pantropical \\
\hline Lecanora caesiorubella Ach. & $\mathrm{RS}$ & $\mathrm{x}$ & $\mathrm{x}$ & Pantropical \\
\hline \multicolumn{5}{|l|}{ Monoblastiaceae } \\
\hline Anisomeridium tamarindii (Fée) R. C. Harris & $\mathrm{RS}$ & $\mathrm{x}$ & $\mathrm{x}$ & Pantropical \\
\hline \multicolumn{5}{|l|}{ Pertusariaceae } \\
\hline Pertusaria carneola (Eschw.) Müll. Arg. & $\mathrm{RS}$ & $\mathrm{x}$ & & Pantropical \\
\hline Pertusaria wulfenioides De Lesd. & $\mathrm{RS}$ & & $\mathrm{x}$ & $\begin{array}{c}\text { Endêmica para o Sudoeste } \\
\text { da América do Norte }\end{array}$ \\
\hline
\end{tabular}


Tabela 1. Continuação.

\begin{tabular}{|c|c|c|c|c|}
\hline \multirow{2}{*}{ Família/Espécies } & \multirow{2}{*}{$\begin{array}{c}\text { Nova } \\
\text { ocorrência }\end{array}$} & \multicolumn{2}{|c|}{ Localização } & \multirow{2}{*}{ Distribuição geográfica } \\
\hline & & Porto Alegre & Viamão & \\
\hline \multicolumn{5}{|l|}{ Pilocarpaceae } \\
\hline Malcolmiella vinosa (Eschw.) Kalb \& Lücking & RS & $\mathrm{x}$ & $\mathrm{x}$ & Pantropical \\
\hline \multicolumn{5}{|l|}{ Physciaceae } \\
\hline Cratiria lauricassiae (Fée) Marbach & RS & $\mathrm{x}$ & $\mathrm{x}$ & Subtropical \\
\hline \multicolumn{5}{|l|}{ Pyrenulaceae } \\
\hline Pyrenula mucosa (Vain.) R. C. Harris & Brasil & $\mathrm{x}$ & $\mathrm{x}$ & Pantropical \\
\hline Pyrenula pyrenuloides (Mont.) R. C. Harris & RS & & $\mathrm{x}$ & Pantropical \\
\hline \multicolumn{5}{|l|}{ Ramalinaceae } \\
\hline Bacidia russeola (Kremp.) Zahlbr. & $\mathrm{RS}$ & $\mathrm{x}$ & & Pantropical \\
\hline \multicolumn{5}{|l|}{ Roccellaceae } \\
\hline Bactrospora myriadea (Fée) Egea \& Torrente & RS & & $\mathrm{x}$ & Pantropical, Temperada \\
\hline Enterographa compunctula (Nyl.) Redinger & RS & $\mathrm{x}$ & & Pantropical \\
\hline
\end{tabular}

As espécies citadas como novas ocorrências para o país possuem distribuição geográfica distinta. Graphis dolichographa foi registrada para Austrália e Colômbia (Lücking et al. 2009), Phaeographis intricans para Austrália, Costa Rica, El Salvador, Guiana, Singapura, Venezuela e Estados Unidos e Pyrenula mucosa para Costa Rica e Papua Nova Guiné (GBIF 2009). Os espécimes liquênicos registrados pela primeira vez para o estado do Rio Grande do Sul foram referenciados especialmente para a região Nordeste, litoral de São Paulo e do Paraná, com exceção de. Chapsa cinchonarum que possui novo registro para a região sul e para o RS, tendo distribuição pantropical com amostras coletadas na Colômbia, África e Brasil (Frisch 2006). Coenogonium subdilutum, Enterographa compunctula, Fissurina instabilis, Graphis parallela foram citadas para o estado de Alagoas; Anisomeridium tamarindii, Bactrospora myriadea, Cratiria lauricassiae, Graphis geraensis, G. paraserpens, G. rigidula, G. schiffneri, G. submarginata, Phaeographis punctiformis, Haematomma personii, Lecanora caesiorubella e Pertusaria carneola para Pernambuco; P. wulfenioides para Sergipe; Graphis kakaduensis para Alagoas e Pernambuco; Malcolmiella vinosa em Alagoas, Pernambuco e Sergipe; Pyrenula pyrenuloides para Alagoas, Pernambuco, Sergipe e Rio Grande do Norte (Cáceres 2007). Bacidia russeola foi registrada em Pernambuco e São Paulo (Cáceres 2007, Marcelli 1992). Dal Forno (2009) cita pela primeira vez Graphis archerii para a região litorânea do Paraná.

A freqüência destas espécies nas áreas avaliadas foi diferenciada, sendo que Anisomeriudium tamarindii ocorreu em $86 \%$ dos pontos seguida de Graphis parallela $(66,7 \%)$, Pertusaria carneola (63,3\%); Graphis submarginata e Haematomma personii (50\%); Cratiria lauricassiae (36,7\%); Coenogonium subdilutum (30\%); Graphis kakaduensis e G. schiffneri (23,3\%); G. archerii, G. geraensis e Pyrenula mucosa (20\%); G. dolichographa, C. cinchonarum, Enterographa compunctula, Fissurina. instabilis, Lecanora caesiorubella (10\%); Graphis rigidula, Malcolmiella vinosa e Phaeographis intricans $(6,7 \%)$ e as demais espécies ocorreram em 3,3\% das áreas. Das áreas estudadas, a maior ocorrência $(56,7 \%)$ de espécies foi identificada para a área do Parque Estadual de Itapuã considerada estação controle, porém cabe salientar que esta diferença não foi tão acentuada em relação ao conjunto das estações estudadas, que apresentou na sua totalidade um número de espécies significante, com um total de 144 táxons. Das espécies crostosas citadas neste estudo, A. tamarindii e P. carneola foram consideradas pela primeira vez como bioindicadoras da qualidade do ar, juntamente com outras seis espécies pertencentes ao grupo morfológico crostoso (Käffer et al. em preparação).

Os novos registros destas espécies crostosas para a área estudada ressaltam a importância e abrangência de estudos deste grupo de liquens. Nota-se aqui que a metodologia empregada para o estudo comparativo entre os forófitos das várias áreas analisadas na cidade de Porto Alegre e no Parque Estadual de Itapuã foi essencial para o registro desta diversidade liquênica, anteriormente desconhecida para todo o estado. 


\section{Agradecimentos}

Agradecemos ao Dr. Robert Lücking do Departamento de Botânica do The Field Museum de Chicago, USA, pela ajuda na identificação de algumas espécies crostosas, às acadêmicas de biologia Camila R. Alves, Viviane Pereira e Natália M. Koch pelo auxílio no campo e ao Museu de Ciências Naturais da Fundação Zoobotânica do Rio Grande do Sul por oferecer as dependências para as atividades de laboratório.

\section{Referências Bibliográficas}

Ahmadjian, V. 1993. The Lichen Symbiosis. New York, John Wiley \& Sons Inc.

Aptroot, A.; Lücking, R.; Sipman, H.J.M.; Umaña, L. \& Chaves, J.L. 2008. Pyrenocarpous lichens with bitunicate asci A first assessment of the lichen biodiversity inventory in Costa Rica. Bibliotheca Lichenologica 97: 1-163.

Cáceres, M.E. da S. 2007. Corticolous crustose and microfoliose lichens of northeastern Brazil. Libri Botanici 22: 1-168.

Dal Forno, M. 2009. A Família Graphidaceae (Ascomycota Liquenizados) em Restinga em Pontal do Sul, Pontal do Paraná, Paraná Dissertação (Mestrado). Universidade Federal do Paraná, Curitiba.

Frisch, A. 2006. Contributions towards a new systematics of the lichen family Thelotremataceae. Dissertation (Zur Erlangung Des Doktorgrades Der Naturwissenschaften). Biologie und Vorklinische Medizin der Universität Regensburg, Bavária.

GBIF - Global Biodiversity Information Facility 2009. Version 1.2.6. http://data.gbif.org/html (Acesso em 01/10/2009).

Livi, F.P. 1998. Elementos do clima: o contraste de tempos frios e quentes. Pp.73-78. In: Menegat, R. (Coord.). Atlas Ambiental de Porto Alegre. Porto Alegre, Ed. da Universidade/ UFRGS.

Lücking, R.; Chaves, J.L.; Sipman, H.J.M.; Umanã, L. \& Aptroot, A. 2008. A first assessment of the Ticolichen biodiversity inventory in
Costa Rica: The genus Graphis, with notes on the genus Hemithecium (Ascomycota: Ostropales: Graphidaceae). Fieldiana Botany 46:1-140.

Lücking, R.; Archer, A.W.\& Aptroot009. A world-wide key to the genus Graphis (Ostropales: Graphidaceae). The Lichenologist 41:1-90.

Lücking, R. \& Rivas-Plata, E. 2008. Clave y Guía Ilustrada Para Géneros de Graphidaceae. Glalia [S.l.] 1: 1-39.

Marcelli, M.P. 1992. Ecologia Liquênica nos Manguezais do Sul-Sudeste Brasileiro. Bibliotheca Lichenologica 47: 1-310.

Marcelli, M.P. 2006. Fungos Liquenizados. Pp 23-74. In: L.X. Filho; M.E. Legaz, C.V \& Córdoba, E.C. Pereira. Biologia de Liquens. Rio de Janeiro, Âmbito cultural.

Marcelli, M.P. Checklist of lichens and lichenicolous fungi from Brazil. Preliminary Version 1. www.unI-hamburg.de/biologie/ialb/herbar/ brazi_f2.html (Acesso em 27/08/2009).

Martins, S.M. de A. 2006. Estudo da comunidade liquenizada epífita em Dodonaea viscosa L. na restinga do Parque Estadual de Itapuã, Viamão, RS. Tese (Doutorado). Instituto de Botânica da Secretaria do MeioAmbiente. São Paulo.

Nash III, T.H. 1996. Introduction. Pp. 1-7. In: Nash III, T.H. Lichen Biology. Cambridge, University Press.

Rivas Plata, E.; Lücking R.; Sipman, H.J.M.; Mangold, A.; Kalb K. \& Lumbsch, T.H. 2009. A world-wide key to the thelotremoid Graphidaceae, excluding the Ocellularia-Myriotrema-Stegobolus clade. The Lichenologist 42: 139-185.

Spielmann, A.A. 2006. Checklist of Lichens Lichenicolous Fungi of Rio Grande do Sul (Brazil). Caderno de Pesquisa Série Biologia 18(2): 7-125.

Sipman, H. Provisional determination keys for the Graphidales of Costa Rica. Disponível em: <http://www.bgbm.org/BGBM/STAFF/ Wiss/ Sipman/Zschackia/ Diorygma/intro.htm (Acesso em 12/12/2006).

Vieira, E.F. 1984. Rio Grande do Sul: geografia física e vegetação. Porto Alegre, Sagra.

Versão eletrônica do artigo em www.scielo.br/abb e http://www.botanica.org.br/acta/ojs 